\title{
A two-level Schwarz preconditioner for heterogeneous problems
}

\author{
V. Dolean ${ }^{1}$, F. Nataf ${ }^{2}$, R. Scheichl ${ }^{3}$ and N. Spillane ${ }^{2}$
}

\section{Introduction}

Coarse space correction is essential to achieve algorithmic scalability in domain decomposition methods. Our goal here is to build a robust coarse space for Schwarz-type preconditioners for elliptic problems with highly heterogeneous coefficients when the discontinuities are not just across but also along subdomain interfaces, where classical results break down $[3,6,15,9]$.

In previous work, [7], we proposed the construction of a coarse subspace based on the low-frequency modes associated with the Dirichlet-to-Neumann (DtN) map on each subdomain. A rigorous analysis was recently provided in [2]. Similar ideas to build stable coarse spaces, based on the solution of local eigenvalue problems on entire subdomains, can be found in [4], and even traced back to similar ideas for algebraic multigrid methods in [1]. However, we will argue below that the DtN coarse space presented here is better designed to deal with coefficient variations that are strictly interior to the subdomain, being as robust as, but leading to a smaller dimension than the coarse space analysed in [4].

The robustness result that we obtain, generalizes the classical estimates for overlapping Schwarz methods to the case where the coarse space is richer than just the constant mode per domain [8], or other classical coarse spaces (cf. [15]). The analysis is inspired by that in $[4,13]$ and crucially uses the framework of weighted Poincaré inequalities, introduced in $[12,10]$ and successfully applied also to other methods in $[11,14]$.

Laboratoire J.A. Dieudonné, CNRS UMR 6621, 06108 Nice Cedex 02, France. dolean@unice.fr · Laboratoire J.L. Lions, CNRS UMR 7598, Université Pierre et Marie Curie, 75005 Paris, France. nataf@ann.jussieu.fr,spillane@ann.jussieu.fr · Department of Mathematical Sciences, University of Bath, Bath BA2 7AY, United Kingdom, R.Scheichl@maths.bath.ac.uk 


\section{Two-level Schwarz method with DtN coarse space}

We consider the variational formulation of a second order, elliptic boundary value problem with Dirichlet boundary conditions: Find $u^{*} \in H_{0}^{1}(\Omega)$, for a given domain $\Omega \subset \mathbb{R}^{d}\left(d=2\right.$ or 3 ) and a source term $f \in L_{2}(\Omega)$, such that

$$
a\left(u^{*}, v\right) \equiv \int_{\Omega} \alpha(x) \nabla u^{*} \cdot \nabla v=\int_{\Omega} f v \equiv(f, v), \quad \forall v \in H_{0}^{1}(\Omega),
$$

and the diffusion coefficient $\alpha=\alpha(x)$ is a positive piecewise constant function that may have large variations within $\Omega$.

We consider a discretization of the variational problem (1) with continuous, piecewise linear finite elements (FE). For a shape regular, simplicial triangulation $\mathcal{T}_{h}$ of $\Omega$, the standard space of continuous and piecewise linear functions (w.r.t $\mathcal{T}_{h}$ ) is then denoted by $V_{h}$. The subspace of functions from $V_{h}$ that vanish on the boundary of $\Omega$ is denoted by $V_{h, 0}$. The discrete FE problem that we want to solve is: Find $u_{h} \in V_{h, 0}$ such that

$$
a\left(u_{h}, v_{h}\right)=\left(f, v_{h}\right), \quad \forall v_{h} \in V_{h, 0} .
$$

Given the usual nodal basis $\left\{\phi_{i}\right\}_{i=1}^{n}$ for $V_{h, 0}$ consisting of "hat" functions with $n:=\operatorname{dim}\left(V_{h, 0}\right),(2)$ can be compactly written as

$$
A \mathbf{u}=\mathbf{f}, \quad \text { with } \quad A_{i j}:=a\left(\phi_{j}, \phi_{i}\right) \text { and } f_{i}=\left(f, \phi_{i}\right), i, j=1, \ldots, n,
$$

where $\mathbf{u}$ and $\mathbf{f}$ are respectively the vector of coefficients corresponding to the unknown FE function $u_{h}$ in (2) and to the r.h.s function $f$.

Two-level Schwarz type methods for (2) are now constructed by choosing an overlapping decomposition $\left\{\Omega_{j}\right\}_{j=1}^{J}$ of $\Omega$ with a subordinate partition of unity $\left\{\chi_{j}\right\}_{j=1}^{J}$, as well as a suitable coarse subspace $V_{H} \subset V_{h, 0}$. In practice the overlapping subdomains $\Omega_{j}$ can be constructed automatically given the system matrix $A$ by using a graph partitioner, such as METIS, and adding on a number of layers of fine grid elements to the resulting nonoverlapping subdomains. A suitable partition of unity can be constructed from the geometric information of the fine grid. For more details see e.g. [15] or [2]. We assume that each point $x \in \Omega$ is contained in at most $N_{0}$ subdomains $\Omega_{j}$.

The crucial ingredient to obtain robust two-level methods for problems with heterogeneous coefficients is the choice of coarse space $V_{H} \subset V_{h, 0}$. Let us assume for the moment that we have such a space $V_{H}$ and a restriction operator $R_{0}$ from $V_{h, 0}$ to $V_{H}$ and define restriction operators $R_{j}$ from functions in $V_{h, 0}$ to functions in $V_{h, 0}\left(\Omega_{j}\right)$, or from vectors in $\mathbb{R}^{n}$ to vectors in $\mathbb{R}^{\operatorname{dim} V_{h, 0}\left(\Omega_{j}\right)}$, by setting $\left(R_{j} u\right)\left(x_{i}\right)=u\left(x_{i}\right)$ for every grid point $x_{i} \in \Omega_{j}$. The two-level overlapping additive Schwarz preconditioner for (3) is then simply

$$
M_{A S, 2}^{-1}=\sum_{j=0}^{J} R_{j}^{T} A_{j}^{-1} R_{j} \quad \text { where } \quad A_{j}:=R_{j} A R_{j}^{T}, j=0, \ldots, J .
$$


In the classical algorithm $V_{H}$ consists simply of FEs on a coarser triangulation $\mathcal{T}_{H}$ of $\Omega$ and $R_{H}$ is the canonical restriction from $V_{h, 0}$ to $V_{H}$, leading to a fully scalable iterative method with respect to mesh/problem size (provided the overlap size is proportional to the coarse mesh size $H$ ). However, unfortunately this preconditioner is not robust to strong variations in the coefficient $\alpha$. We will now present a new, completely local approach to construct a robust coarse space, as well as an associated restriction operator using eigenvectors of local Dirichlet-to-Neumann maps, proposed in [7].

We start by constructing suitable local functions on each subdomain $\Omega_{j}$ that will then be used to construct a basis for $V_{H}$. To this end, let us fix $j \in$ $\{1, \ldots, J\}$ and first consider at the continuous level the Dirichlet-to-Neumann map $\operatorname{DtN}_{j}$ on the boundary of $\Omega_{j}$. Let $\Gamma_{j}:=\partial \Omega_{j}$ and let $v_{\Gamma}: \Gamma_{j} \rightarrow \mathbb{R}$ be a given function, such that $\left.v_{\Gamma}\right|_{\partial \Omega}=0$ if $\Gamma_{j} \cap \partial \Omega \neq \emptyset$. We define

$$
\operatorname{DtN}_{j}\left(v_{\Gamma}\right):=\left.\alpha \frac{\partial v}{\partial \nu_{j}}\right|_{\Gamma_{j}}
$$

where $\nu_{j}$ is the unit outward normal to $\Omega_{j}$ on $\Gamma_{j}$, and $v$ satisfies

$$
-\operatorname{div}(\alpha \nabla v)=0 \text { in } \Omega_{j}, \quad v=v_{\Gamma} \text { on } \Gamma .
$$

The function $v$ is the $\alpha$-harmonic extension of the boundary data $v_{\Gamma}$ to the interior of $\Omega_{j}$.

To construct the (local) coarse basis functions, we now find the low frequency modes of the Dirichlet-to-Neumann operator $\mathrm{DtN}_{j}$ with respect to the weighted $L_{2}-$ norm on $\Gamma_{j}$, i.e. the smallest eigenvalues of

$$
\operatorname{DtN}_{j}\left(v_{\Gamma}^{(j)}\right)=\lambda^{(j)} \alpha v_{\Gamma}^{(j)}
$$

Then we extend each of these modes $v_{\Gamma}^{(j)} \alpha$-harmonically to the whole domain and let $v^{(j)}$ be its extension. This is equivalent to the Steklov eigenvalue problem of looking for the pair $\left(v^{(j)}, \lambda^{(j)}\right)$ which satisfies:

$$
-\operatorname{div}\left(\alpha \nabla v^{(j)}\right)=0 \text { in } \Omega_{j} \quad \text { and } \quad \alpha \frac{\partial v^{(j)}}{\partial \nu_{j}}=\lambda \alpha v^{(j)} \text { on } \Gamma_{j}
$$

The variational formulation of $(7)$ is to find $\left(v^{(j)}, \lambda^{(j)}\right) \in H^{1}\left(\Omega_{j}\right) \times \mathbb{R}$ such that

$$
\int_{\Omega_{j}} \alpha \nabla v^{(j)} \cdot \nabla w=\lambda^{(j)} \int_{\Gamma_{j}} \operatorname{tr}_{j} \alpha v^{(j)} w, \quad \forall w \in H^{1}\left(\Omega_{j}\right),
$$

where $\operatorname{tr}_{j} \alpha(x):=\lim _{y \in \Omega_{j} \rightarrow x} \alpha(y)$. To discretize this generalized eigenvalue problem, we consider for all $v, w \in H^{1}\left(\Omega_{j}\right)$ the bilinear forms

$$
a_{j}(v, w):=\int_{\Omega_{j}} \alpha \nabla v \cdot \nabla w \text { and } m_{j}(v, w):=\int_{\Gamma_{j}} \operatorname{tr}_{j} \alpha v w
$$


and restrict (8) to the FE space $V_{h}\left(\Omega_{j}\right)$. The coefficient matrices associated with the variational forms $a_{j}$ and $m_{j}$ are

$$
A_{k l}^{(j)}:=\int_{\Omega_{j}} \alpha \nabla \phi_{k} \cdot \nabla \phi_{l} \quad \text { and } \quad M_{k l}^{(j)}:=\int_{\Gamma_{j}} \operatorname{tr}_{j} \alpha \phi_{k} \phi_{l},
$$

where $\phi_{k}$ and $\phi_{l}$ are any two nodal basis functions for $V_{h}\left(\Omega_{j}\right)$ associated with vertices of $\mathcal{T}_{h}$ contained in $\bar{\Omega}_{j}$. Then the FE approximation to (8) in matrix notation is

$$
A^{(j)} \mathbf{v}^{(j)}=\lambda^{(j)} M^{(j)} \mathbf{v}^{(j)}
$$

where $\mathbf{v}^{(j)} \in \mathbb{R}^{n_{j}}, n_{j}:=\operatorname{dim} V_{h}\left(\Omega_{j}\right)$, denotes the degrees of freedom of the FE approximation to $v^{(j)}$ in $V_{h}\left(\Omega_{j}\right)$.

Let the $n_{j}$ eigenpairs $\left(\lambda_{\ell}^{(j)}, \mathbf{v}_{\ell}\right)_{\ell=1}^{n_{j}}$ corresponding to (9) be numbered in increasing order of $\lambda_{\ell}^{(j)}$. Since $M_{k l}^{(j)} \neq 0$ only if $\phi_{k}$ and $\phi_{l}$ are associated with the $n_{\Gamma}$ vertices of $\mathcal{T}_{h}$ that lie on $\Gamma_{j}$, it is easy to see that at most $n_{\Gamma}$ of the eigenvalues $\lambda_{\ell}^{(j)}$ are finite. Moreover, the smallest eigenvalue $\lambda_{1}^{(j)}=0$ with constant eigenvector and the set of eigenvectors $\left\{\mathbf{v}_{\ell}\right\}_{\ell=1}^{n_{j}}$ can be chosen so that they are $A^{(j)}$-orthonormal. The local coarse space is now defined as the span of the FE functions $v_{\ell}^{(j)} \in V_{h}\left(\Omega_{j}\right), \ell \leq m_{j} \leq n_{\Gamma}$, corresponding to the first $m_{j}$ eigenpairs of (9). For each subdomain $\Omega_{j}$, we choose the value of $m_{j}$ such that $\lambda_{\ell}^{(j)}<\operatorname{diam}\left(\Omega_{j}\right)^{-1}$, for all $\ell \leq m_{j}$, and $\lambda_{m_{j}+1}^{(j)} \geq \operatorname{diam}\left(\Omega_{j}\right)^{-1}$. We will see in the analysis in the next section why this is a sensible choice.

Using the partition of unity $\left\{\chi_{j}\right\}_{j=1}^{J}$, we now combine the local basis functions constructed in the previous section to obtain a conforming coarse space $V_{H} \subset V_{h, 0}$ on all of $\Omega$. The new coarse space is defined as

$$
V_{H}:=\operatorname{span}\left\{I_{h}\left(\chi_{j} v_{\ell}^{(j)}\right): 1 \leq j \leq J \text { and } 1 \leq \ell \leq m_{j}\right\}
$$

where $I_{h}$ is the standard nodal interpolant onto $V_{h, 0}(\Omega)$. The dimension of $V_{H}$ is $\sum_{j=1}^{J} m_{j}$. By construction each of the functions $I_{h}\left(\chi_{j} v_{\ell}^{(j)}\right) \in V_{h_{0}}$, so that as required $V_{H} \subset V_{h, 0}$. The transfer operator $R_{0}$ from $V_{h_{0}}$ to $V_{H}$ is defined in a canonical way by setting $R_{0}^{T} u_{H}\left(x_{i}\right)=u_{H}\left(x_{i}\right)$, for all $u_{H} \in V_{H}$ and for all vertices $x_{i}$ of $\mathcal{T}_{h}$.

We will see in the next section that under some mild assumptions on the variability of $\alpha$ this choice of coarse space leads to a scalable and coefficientrobust domain decomposition method with supporting theory.

\section{Conditioning analysis}

To analyse this method let us first define the boundary layer $\Omega_{j}^{\circ}$ for each $\Omega_{j}$ that is overlapped by neighbouring domains, i.e. 


$$
\Omega_{j}^{\circ}:=\left\{x \in \Omega_{j}: \chi_{j}(x)<1\right\} .
$$

We assume that this layer is uniformly of width $\geq \delta_{j}$, in the sense that it can be subdivided into shape regular regions of diameter $\delta_{j}$, and that the triangulation $\mathcal{T}_{h}$ resolves it. This also guarantees that it is possible to find a partition of unity such that $\left|\chi_{j}\right|=\mathcal{O}(1)$ and $\left|\nabla \chi_{j}\right|=\mathcal{O}\left(\delta_{j}^{-1}\right)$.

We now state the key assumption on the coefficient distribution $\alpha(x)$.

Assumption 1 We assume that, for each $j=1, \ldots, J$, there exists a set $X_{j} \subset \Gamma_{j}$ (not necessarily connected) such that (i) $\max _{x, y \in X_{k}} \frac{\alpha(x)}{\alpha(y)}=\mathcal{O}(1)$ and (ii) there exists a path $P_{y}$ from each $y \in \Omega_{j}$ to $X_{j}$, such that $\alpha(x)$ is an increasing function along $P_{y}$ (from $y$ to $X_{j}$ ).

Lemma 1 (weighted Poincaré inequality [10]). Let Assumption 1 hold.

$$
\int_{\Omega_{j}^{\circ}} \alpha\left|v-\bar{v}^{X_{j}}\right|^{2} \leq C_{P} \delta_{j} \int_{\Omega_{j}^{\circ}} \alpha|\nabla v|^{2}, \quad \text { for all } v \in V_{h}\left(\Omega_{j}\right),
$$

where $\bar{v}^{X_{j}}:=\frac{1}{\left|X_{j}\right|} \int_{X_{j}} v$.

Remark 1. Note that Assumption 1 is related to the classical notion of quasimonotonicity coined in [3]. It ensures that the constant $C_{P}$ in the Poincarétype inequality in Lemma 1, as well as all the other (hidden) constants below are independent of the values of the coefficient function $\alpha(x)$. The constants may however depend logarithmically or linearly on $\delta_{j} / h$. This depends on the geometry and shape of the paths $P_{y}$ and on the size and shape of the set $X_{j}$. For more details see $[2]$ and $[12,10]$.

The following proposition is the central result in our analysis. It proves the stability and a weak approximation property for a local projection onto the span of the first $m_{j}$ eigenvectors.

Proposition 1. Let Assumption 1 hold, and for any $u \in V_{h}\left(\Omega_{j}\right)$, define the projection $\Pi_{j} u:=\sum_{\ell=1}^{m_{j}} a_{j}\left(v_{\ell}^{(j)}, u\right) v_{\ell}^{(j)}$. Then

$$
\begin{aligned}
\left|\Pi_{j} u\right|_{a, \Omega_{j}} & \leq|u|_{a, \Omega_{j}} \text { and } \\
\left\|u-\Pi_{j} u\right\|_{0, \alpha, \Omega_{j}^{\circ}} & \lesssim \sqrt{c_{j}\left(m_{j}\right)} \delta_{j}|u|_{a, \Omega_{j}} .
\end{aligned}
$$

where $c_{j}\left(m_{j}\right):=C_{P}^{2}+\left(\delta_{j} \lambda_{m_{j}+1}^{(j)}\right)^{-1}$.

Proof. Theorem 3.2 in [2].

As usual (cf. [15]), the following condition number bound can then be obtained via abstract Schwarz theory by constructing a stable splitting.

Theorem 2. Let Assumption 1 be satisfied. Then the condition number of the two-level Schwarz algorithm with the coarse space $V_{H}$ based on local DtN maps and defined in (10) can be bounded by 


$$
\kappa\left(M_{A S, 2}^{-1} A\right) \lesssim \max _{j=1}^{J}\left\{c_{j}\left(m_{j}\right)\right\} \lesssim C_{P}^{2}+\max _{j=1}^{J}\left(\delta_{j} \lambda_{m_{j}+1}^{(j)}\right)^{-1} .
$$

The hidden constant is independent of $h, \delta_{j}, \operatorname{diam}\left(\Omega_{j}\right)$, and $\alpha$.

Proof. This is Theorem 3.5 in [2]. The stable splitting for a function $u \in V_{h, 0}$ is constructed using the projections $\Pi_{j}, j=1, \ldots, J$, in Proposition 1 to define the coarse quasi-interpolant

$$
u_{0}:=I_{h}\left(\left.\sum_{j=1}^{J} \chi_{j} \Pi_{j} u\right|_{\Omega_{j}}\right) \in V_{H}
$$

If we now choose $u_{j}:=I_{h}\left(\chi_{j}\left(u-\Pi_{j} u\right)\right) \in V_{h, 0}\left(\Omega_{j}\right)$, then

$$
u=\sum_{j=0}^{J} u_{j} \quad \text { and } \quad \sum_{j=0}^{J} \int_{\Omega} \alpha\left|\nabla u_{j}\right|^{2} \lesssim \max _{j=1}^{J}\left\{c_{j}\left(m_{j}\right)\right\} \int_{\Omega} \alpha|\nabla u|^{2}
$$

For details see [2].

Remark 2. Note that by choosing the number $m_{j}$ of modes per subdomain such that $\lambda_{m_{j}+1}^{(j)} \geq \operatorname{diam}\left(\Omega_{j}\right)^{-1}$, as stated in Section 2, we have

$$
\kappa\left(M_{A S, 1}^{-1} A\right) \lesssim\left(C_{P}^{2}+\max _{j=1}^{J} \frac{\operatorname{diam}\left(\Omega_{j}\right)}{\delta_{j}}\right) .
$$

Hence, provided the constant $C_{P}$ is uniformly bounded, independently of any jumps in the coefficients, we retrieve the classical estimate for the twolevel additive Schwarz method independently of any variations of coefficients across or along subdomain boundaries.

\section{Numerical results}

We choose $\Omega=(0,1)^{2}$ and discretize (1) on regular grid with $m \times m$ elements. We apply a homogeneous Dirichlet boundary condition $u=0$ on the left hand side boundary and homoegenous Neumann boundary conditions $\frac{\partial u}{\partial \nu}=0$ on the remainder. We use the METIS partitioner to split the domain into 16 irregular subdomains as shown in Figure 1. Then we construct the overlapping partition using Freefem $++[5]$ by extending each subdomain by one layer of elements on all or the boundary.

As the coarse space we use the DtN coarse space described in Section 2 with $m_{j}$ chosen such that $\lambda_{m_{j}}^{(j)}<\operatorname{diam}\left(\Omega_{j}\right)^{-1} \leq \lambda_{m_{j}+1}^{(j)}$, for all $j=1, \ldots, 16$ (labelled D2N below). We compare this preconditioner with the one-level additive Schwarz method (labelled NONE below) and the two-level method with 
partition of unity coarse space, i.e. choosing $m_{j}=1$, for all $j=1, \ldots, 16$ (labelled POU below). To confirm in some sense the optimality of our choice for $m_{j}$, we also include results with the DtN coarse space choosing $m_{j}+1$ and $\max \left\{1, m_{j}-1\right\}$ basis functions per subdomain (labelled D2N+ and D2N-, respectively). We use the preconditioners within a conjugate gradient iteration and terminate when the residual has been reduced by a factor $10^{-6}$.

In the first test case, we choose $m=80$ and $\alpha$ to be a realization of a lognormal distribution with exponential covariance function (variance $\sigma^{2}=4$ and correlation length $\lambda=4 / m)$ and mean $(\log \alpha)=3$ (cf. Figure ??).

In Figure 3 we plot $\|u-\bar{u}\|_{\infty}$ (where $\bar{u}$ is the solution of (3) obtained via a direct solver) against the iteration count. We compare three methods:

AS : the one level preconditioner $M_{A S, 1}^{-1}=\sum_{j=1}^{J} R_{j}^{T} A_{j}^{-1} R_{j}$.

$\mathrm{AS}+\mathrm{Z}_{\mathrm{NICO}}$ : the two level preconditioner defined by (4) with a coarse grid which consists simply of constant functions on each subdomain weighted by a partition of unity.

$\mathrm{AS}+\mathrm{Z}_{\mathrm{D} 2 \mathrm{~N}}$ : the two level preconditioner defined by (4) with the new coarse grid we have introduced.

The $\mathrm{AS}$ and $\mathrm{AS}+\mathrm{Z}_{\mathrm{NICO}}$ methods require roughly the same number of iterations (89 versus 92 iterations) whereas the new $\mathrm{AS}+\mathrm{Z}_{\mathrm{D} 2 \mathrm{~N}}$ stands out reducing the number of iterations to 38 . Finally, in Figure 4 we show that the criterion for the number $m_{j}$ of eigenmodes that we select in each subdomain is somewhat optimal since adding one has hardly any impact on performance while removing one has strong negative impact.

\section{References}

[1] T. Chartier, R. D. Falgout, V. E. Henson, J. Jones, T. Manteuffel, S. McCormick, J. Ruge, and P. S. Vassilevski. Spectral AMGe ( $\rho$ AMGe). SIAM J. Sci. Comput., 25(1):1-26, 2003.

[2] V. Dolean, F. Nataf, Scheichl R., and N. Spillane. Analysis of a twolevel Schwarz method with coarse spaces based on local Dirichlet-toNeumann maps. http://hal.archives-ouvertes.fr/hal-00586246/fr/, 2011.

[3] M. Dryja, M. V. Sarkis, and O. B. Widlund. Multilevel Schwarz methods for elliptic problems with discontinuous coefficients in three dimensions. Numer. Math., 72(3):313-348, 1996.

[4] J. Galvis and Y. Efendiev. Domain decomposition preconditioners for multiscale flows in high contrast media: Reduced dimension coarse spaces. Multiscale Modeling \& Simulation, 8(5):1621-1644, 2010.

[5] Frédéric Hecht. FreeFem++. Numerical Mathematics and Scientific Computation. Laboratoire J.L. Lions, Université Pierre et Marie Curie, http://www.freefem.org/ff++/, 3.7 edition, 2010. 


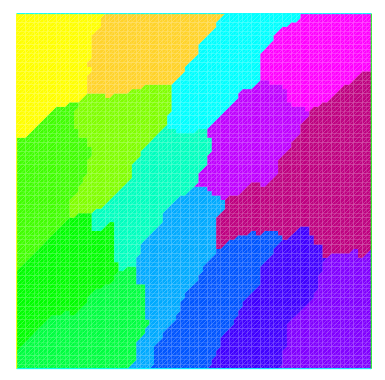

Fig. 116 subdomains

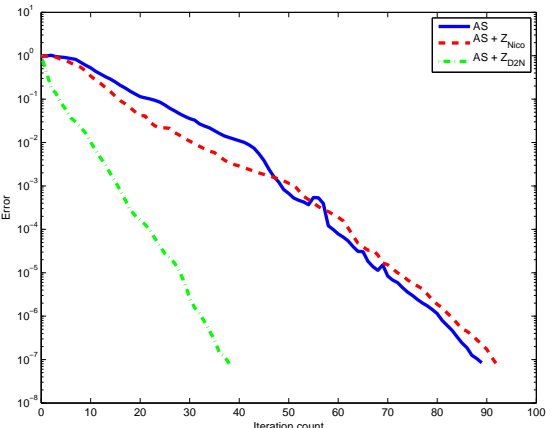

Fig. 3 Convergence

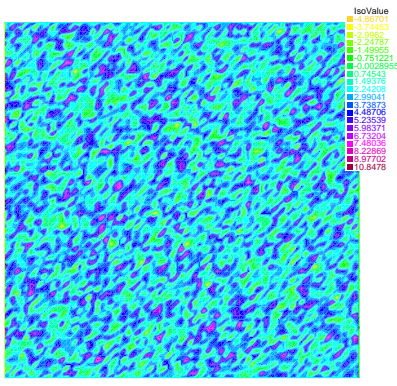

Fig. $2 \log (\alpha)\left(0<\alpha<5 \cdot 10^{7}\right)$

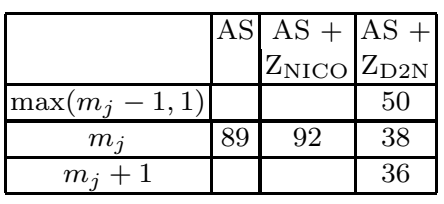

Fig. 4 Optimality of the criterion - Number of iterations needed when we add or remove one mode per subdomain as compared to the number $m_{j}$ given by the automatic method

[6] J. Mandel and M. Brezina. Balancing domain decomposition for problems with large jumps in coefficients. Math. Comp., 65:1387-1401, 1996.

[7] F. Nataf, H. Xiang, and V. Dolean. A two level domain decomposition preconditioner based on local Dirichlet-to-Neumann maps. C. $R$. Mathématique, 348(21-22):1163-1167, 2010.

[8] R. A. Nicolaides. Deflation of conjugate gradients with applications to boundary value problems. SIAM J. Numer. Anal., 24(2):355-365, 1987.

[9] C. Pechstein and R. Scheichl. Scaling up through domain decomposition. Appl. Anal., 88(10-11):1589-1608, 2009.

[10] C. Pechstein and R. Scheichl. Weighted Poincaré inequalities. Technical Report NuMa-Report 2010-10, Institute of Computational Mathematics, Johannes Kepler University, Linz, December 2010. submitted.

[11] C. Pechstein and R. Scheichl. Analysis of FETI methods for multiscale PDEs - Part II: Interface variation. Numer. Math., 2011. Published online 21 February 2011.

[12] C. Pechstein and R. Scheichl. Weighted Poincaré inequalities and applications in domain decomposition. In Y. Huang, R. Kornhuber, O. Widlund, and J. Xu, editors, Domain Decomposition Methods in Science and Engineering XIX, volume 78 of LNCSE, pages 197-204. Springer, 2011. 
[13] R. Scheichl, P. S. Vassilevski, and L. T. Zikatanov. Weak approximation properties of elliptic projections with functional constraints. Technical Report LLNL-JRNL-462079, Lawrence Livermore National Lab, 2011.

[14] R. Scheichl, P.S. Vassilevski, and L.T. Zikatanov. Multilevel methods for elliptic problems with highly varying coefficients on non-aligned coarse grids. SIAM J Numer Anal, 2011. Accepted subject to minor corrections.

[15] A. Toselli and O. B. Widlund. Domain decomposition methods - algorithms and theory. Springer, Berlin, 2005. 